\title{
Which group velocity of light in a dispersive medium?
}

\author{
Omar El Gawhary \\ Optics Research Group, Department of Imaging \\ Science and Technology, Delft University of Technology, \\ Lorentzweg 1, 2628 CJ Delft, The Netherlands \\ Sergio Severini \\ Centro Interforze Studi per le Applicazioni Militari \\ Via della Bigattiera 10, 56122 San Piero a Grado (Pi), Italy \\ Paolo Christillin \\ Dipartimento di Fisica, Università di Pisa \\ Largo Bruno Pontecorvo 3, 56127 Pisa, Italy
}

(Dated: November 4, 2018)

\begin{abstract}
The interaction between a light pulse, traveling in air, and a generic linear, non-absorbing and dispersive structure is analyzed. It is shown that energy conservation imposes a constraint between the group velocities of the transmitted and reflected light pulses. It follows that the two fields propagate with group velocities depending on the dispersive properties of the environment (air) and on the transmission properties of the optical structure, and are one faster and the other slower than the incident field. In other words, the group velocity of a light pulse in a dispersive medium is reminiscent of previous interactions. One example is discussed in detail.
\end{abstract}

PACS numbers: 42.25.-p, 78.20.Ci, 11.30.-j 
The concept of group velocity of an electromagnetic wave packet, born in the early $19^{\text {th }}$ century [1], has been thoroughly analyzed in many propagation regimes: for dispersive, nondispersive and absorbing properties of the propagation environment [2]. Such a quantity is naturally linked to the propagation velocity of energy. In fact, if the optical field propagates in dispersive and non-absorbing media, the two concepts of group and energy velocity tend to coincide [1]. In recent years this topic has induced a good deal of studies mainly within the context of the superluminal behavior of light, where the role of several phenomena, like material gain, Kerr nonlinearities, birifrangence, has been examined in detail [3]. Apart from all these interesting results, in our opinion there still exists some simple, but intriguing, aspect in this scenario that deserves to be studied. Actually, in the present Letter we consider a wave packet incident over a generic dispersive, non-absorbing optical structure like a dielectric slab, a Beam Splitter or a finite Photonic Band Gap (PBG) etc.. On general grounds, due to the interaction of the wave packet with this optical structure, we have two emerging fields: the transmitted and the reflected pulse (as schematically depicted in Fig.11). We tackle the problem of their group velocities. As above mentioned, we will only assume that the propagation environment is dispersive, like air is, and that the involved field frequencies are far from the absorbtion lines. It will be shown that, due to the combination of the principle of energy conservation, the dispersive properties of the propagation environment and the transmission properties of the generic optical structure, differences among the group velocities of incident, transmitted and reflected fields arise. For the sake of simplicity, and without loss of generality, we will refer only to fields having the wave vector $\mathbf{k}$ lying in the $x, y$ plane, as shown in Fig 1. We can expand the electric field $E^{(i)}(x, y, t)$ of the incident wave packet, in plane waves:

$$
E^{(i)}(x, y, t)=\frac{1}{2 \pi} \int E^{(i)}(\omega) \exp \left[i\left(\mathbf{k}^{(i)} \cdot \mathbf{r}-\omega t\right)\right] d \omega
$$

where $\mathbf{r}=(x, y)$. In Eq.1 $\mathbf{k}^{(i)}=k(\omega) \mathbf{s}^{(i)}$, where $\mathbf{s}^{(i)}=(\cos \theta,-\sin \theta)$ and $\theta$ is the angle of incidence, and

$$
k(\omega)=n(\omega) \omega / c .
$$

In Eq, $2, n(\omega)$ is the refractive index of the environment which in nonabsorbing media is a real function of a real variable. As usual, the input-output behavior of a generic structure, with respect to the incident field, is describable by means of reflection and transmission coefficients, $r(\omega)$ and $t(\omega)$ respectively, connected with the (intensity) reflectivity and trans- 
missivity through the well known relationships $R(\omega)=|r(\omega)|^{2}$ and $T(\omega)=|t(\omega)|^{2}$. Under such hypotheses [7], [8], [9]

$$
T(\omega)+R(\omega)=|t(\omega)|^{2}+|r(\omega)|^{2}=1
$$

The transmitted and reflected beams are

$$
\begin{aligned}
E^{(t)}(x, y, t) & =\frac{1}{2 \pi} \int t(\omega) E^{(i)}(\omega) \\
& \left.\times \exp \left[i \mathbf{k}^{(t)} \cdot \mathbf{r}-\omega t\right)\right] d \omega \\
E^{(r)}(x, y, t) & =\frac{1}{2 \pi} \int r(\omega) E^{(i)}(\omega) \\
& \times \exp \left[i\left(\mathbf{k}^{(r)} \cdot \mathbf{r}+\omega t\right)\right] d \omega
\end{aligned}
$$

where $\mathbf{k}^{(r)}=k(\omega) \mathbf{s}^{(r)}, \mathbf{k}^{(t)}=k(\omega) \mathbf{s}^{(t)}$ and $\mathbf{s}^{(r)}=(-\cos \theta,-\sin \theta)$ and $\mathbf{s}^{(t)}=(\cos \theta,-\sin \theta)$. Group velocities are defined as

$$
\mathbf{v}_{\mathbf{g}}{ }^{(\alpha)}=\left(\frac{d \omega}{d k}\right)_{k_{0}^{(\alpha)}} \mathbf{s}^{(\alpha)}=\left(\frac{d k}{d \omega}\right)_{\omega_{0}^{(\alpha)}}^{-1} \mathbf{s}^{(\alpha)} .
$$

where $\alpha=\{i, r, t\}$ and $k_{0}^{(\alpha)}=k\left(\omega_{0}^{(\alpha)}\right)$ is the amplitude of the wave vector corresponding to the frequency $\omega_{0}^{(\alpha)}$ at which the spectrum $E^{(\alpha)}(\omega)$ has a maximum in the amplitude. This definition is due to the well known stationary phase method [1]. As we are interested in highlighting the effect of Eq, 3 on group velocities, it is necessary to make some further hypotheses about the spectrum of the incident field as well as on the behavior of $T(\omega)$ and $R(\omega)$. In particular, to exclude interactions producing very distorded wave packets, for which the definition of group velocity would be questionable, we will assume that the spectrum of the incident pulse is narrower than the transmission or reflection bands. This guarantees that the spectral range of the incident pulse is sufficiently limited to include at most one maximum (minimum) of $T(\omega)(R(\omega))$ or vice versa. However, also under such an hypothesis there is some pathological case that must be excluded. This happens when the frequency spectrum of the incident pulse has its maximum exactly at the point where $T(\omega)=1$ (or $R(\omega)=1$ ). In such a case the reflected (transmitted) pulse would be, in practice, either completely absent or very distorted. Generally speaking, if the modulus of the incident field $\left|E^{(i)}(\omega)\right|$ has a maximum (for the intensity of the beams we are obviously interested only in moduli) for a given value of $\omega$ (determinated by the condition $d\left|E^{(i)}(\omega)\right| / d \omega=0$ ), which we denote as $\omega_{0}^{(i)}$, the reflected and transmitted spectra will show a shift in their maxima. 
They will be respectively determined by the following two equations

$$
\begin{aligned}
& \frac{d\left|E^{(i)}(\omega)\right|}{d \omega}|t(\omega)|+\frac{d|t(\omega)|}{d \omega}\left|E^{(i)}(\omega)\right|=0 \\
& \frac{d\left|E^{(i)}(\omega)\right|}{d \omega}|r(\omega)|+\frac{d|r(\omega)|}{d \omega}\left|E^{(i)}(\omega)\right|=0
\end{aligned}
$$

All the three fields would have a maximum in $\omega_{0}^{(i)}$ if, and only if, in that point there is a maximum (or a minimum) for $T(\omega)$ or $R(\omega)$ [1]]. In such a case the fields would have the same group velocity. In all other situations, i.e. when $d T(\omega) /\left.d \omega\right|_{\omega_{0}^{(i)}}=-d R(\omega) /\left.d \omega\right|_{\omega_{0}^{(i)}} \neq 0$ (where $\omega_{0}^{(t)}$ and $\omega_{0}^{(r)}$ denote the solutions of Eqs. 7 and 8 respectively), we can evaluate the moduli for the new group velocities as

$$
\begin{aligned}
& v_{g}^{(t)}=\left(\frac{d \omega}{d k}\right)_{k_{0}^{(t)}}=v_{g}^{(i)}+\left(\frac{d^{2} \omega}{d k^{2}}\right)_{k_{0}^{(i)}}\left(k_{0}^{(t)}-k_{0}^{(i)}\right) \\
& v_{g}^{(r)}=\left(\frac{d \omega}{d k}\right)_{k_{0}^{(r)}}=v_{g}^{(i)}+\left(\frac{d^{2} \omega}{d k^{2}}\right)_{k_{0}^{(i)}}\left(k_{0}^{(r)}-k_{0}^{(i)}\right)
\end{aligned}
$$

where a Taylor expansion has been made. In Eqq. 9 and 10 we have obviously $k_{0}^{(t)} \neq k_{0}^{(r)}$. The reflected and transmitted pulses cannot have same group velocities. This property originates from the observation that, by virtue of the constraint described by the Eq $[3$, spectral changes shown by the two emerging fields are not independent. In fact, it is easily seen that every time $T(\omega)$ grows, in an interval of $\omega$, then in the same interval $R(\omega)$ must necessarily decrease and vice versa. This implies that they must always have different velocities, both different from the group velocity of the incident pulse. The difference between group velocities reported in Eqq. 9, 10, depends on the dispersive properties of the propagation environment (the second derivative term) and on the properties of the optical structure considered (the $k$ 's difference term). Of course in any non-dispersive environment, i.e. when the modulus of wave vector is given by $k=n \omega / c$ with $n$ constant (for instance $n=1$ for the vacuum), the second derivative terms in Eqs. 9-10 are null. Therefore in such a case the above discussed phenomenon is absent.

The competing effect of the group velocity dispersion $\left(\Delta v_{g}\right)$ must also be taken into account:

$$
\Delta v_{g}=\left(\frac{d^{2} \omega}{d k^{2}}\right)_{k_{0}} \Delta k .
$$

It holds for the spread of each single wave packet due to the effect of the dispersive medium. This phenomenon tends to destroy the packet by spreading it while propagating [15]. Hence 
only structures where this effect is under control will be considered.

As an illustration let us calculate the difference for the group velocities of the two emerging pulses in air after an interaction with a dielectric slab. We can evaluate the second derivative term in Eqs. 9, 10 with an accuracy of 1 ppm [13] by using the most accurate recent measurement of the refractive index of air [12] which improves Cauchy's formula for the same refractive index [7], $n(\omega)=1+A\left(1+B \omega^{2}\right)$ with $A=28.79 \times 10^{-5}$ and $B=1.6 \times 10^{-33} s^{2}$. As to the optical structure, we consider a homogeneous, non-dispersive, rectangular dielectric slab with refraction index $n=1.5$ and thickness $d=0.3 \mu m$ along the $x$ axis. The incident field impinging on this structure is supposed to be a broad-spectrum gaussian light pulse (white light), centrered around $0.555 \mu m: E^{(i)}(\omega)=E_{0}^{(i)} \exp \left\{-\left(\omega-\omega_{0}^{(i)}\right)^{2} / 2 \sigma_{0}^{2}\right\}$, with $\omega_{0}^{(i)}=3.39397 \times 10^{15} \mathrm{rad} / \mathrm{s}$ and $\sigma_{0}=4 \times 10^{14} \mathrm{rad} / \mathrm{s}$. It is well known that, in this case, the reflectivity $\mathcal{R}_{\perp}$ for the interface between air/glass depends on the polarization features of the field under study as well as on the (mean) angle of incidence. For the case of orthogonal polarization (electric field perpendicular to the $x, y$ plane) $\mathcal{R}_{\perp}=\sin ^{2}\left(\theta_{i}-\theta_{t}\right) / \sin ^{2}\left(\theta_{i}+\theta_{t}\right)$. Here $\theta_{i}$ and $\theta_{t}$ represent the incident and transmitted angle, respectively. For $\theta_{i}=65^{\circ}$ it follows from Snell's law, $\theta_{t} \cong 37^{\circ}$ and $\mathcal{R}_{\perp} \cong 0.228$. The transmission properties of the entire optical structure are given by

$$
T(\omega)=\frac{1}{1+F \sin ^{2}(\alpha)}
$$

where $F=4 \mathcal{R}_{\perp} /\left(1-\mathcal{R}_{\perp}\right)^{2}, \alpha=\omega n d \cos \left(\theta_{t}\right) / c$ [7]. In Fig 2 (subplot a) we show the frequency spectrum of the incident pulse together with the transmission and reflection spectra of the dielectric slab (which is, after all, a Fabry-Perot multiple beam resonator), while in subplot b we show the frequency spectra for incident, transmitted and reflected fields [14]. It is manifest that the transmitted and reflected pulses are shifted, with respect to the incident one, but in two opposite directions. Indeed we obtain:

$$
\begin{gathered}
v_{g}^{(t)}-v_{g}^{(i)}=2.47 \times 10^{2} \mathrm{~m} / \mathrm{s} \\
v_{g}^{(r)}-v_{g}^{(i)}=-1.65 \times 10^{2} \mathrm{~m} / \mathrm{s}
\end{gathered}
$$

Recalling that the speed of light is known to an accuracy of $\pm 1 \mathrm{~m} / \mathrm{s}$, it is evident that such an effect is observable.

Summarizing, we investigated the role played by energy conservation on the group velocity of a light pulse, propagating in dispersive media, after an interaction with a generic linear, 
non-absorbing and dispersive structure. We have shown that in general, transmitted and reflected fields emerge with different group velocities. In a forthcoming paper the extension of the present formalism to the case of absorptive media will be considered.

[1] M. A. Biot, Phys. Rev., 105, 1129-1137, (1957); Leon Brillouin, "Wave Propagation and Group Velocity", Accademic Press Inc. (London), (1960).

[2] Ashok Puri and Joseph L. Birman, Phys. Rev. Lett., 47, 173-176, (1981); Eric L. Bolda and Raymond Y. Chiao, John C. Garrison, Phys. Rev. A, 48, 3890-3894 (1993).

[3] Alexander A. Govyadinov and Viktor A. Podolskiy, Phys. Rev. Lett., 97, 223902, (2006).

[4] Qiguang Yang, Jae Tae Seo, Bagher Tabibi, and Huitian Wang, Phys. Rev. Lett., 95, 063902, (2005).

[5] Nicolas Brunner, Valerio Scarani, Mark Wegmuller, Matthieu Legre, and Nicolas Gisin, Phys. Rev. Lett., 93, 203902-1, (2004).

[6] Andrea Aiello, Phys. Rev. A, 62, 063813-(14), (2000);

[7] M. Born and E. Wolf, Principles of optics, Cambridge University Press, seventh (expanded) edition, p. 43, (2001).

[8] G. R. Fowles, Introduction to Modern Optics, Dover Publication, Inc., New York, p. 90, (1989).

[9] Stephen M. Barnett, John Jeffers, and Alessandra Gatti, Rodney Loudon, Phys. Rev. A, 57, 2134-2144, (1998).

[10] Eric L. Bolda and Raymond Y. Chiao, John C. Garrison, Phys Rev. A, 48, 3890-3894, (1993)

[11] This condition requires $d T(\omega) /\left.d \omega\right|_{\omega_{0}^{(i)}}=-d R(\omega) /\left.d \omega\right|_{\omega_{0}^{(i)}}=0$ and $d^{2} T(\omega) /\left.d \omega^{2}\right|_{\omega_{0}^{(i)}}=$ $-d^{2} R(\omega) /\left.d \omega^{2}\right|_{\omega_{0}^{(i)}} \neq 0$, in order to exclude cases of horizontal flex points. In addition further requirements on the second order derivatives must be fulfilled to assure that $\omega_{0}^{(i)}$ is a maximum for both fields, and not for only one of them. For instance, if in $\omega_{0}^{(i)}$ there is a maximum of $T(\omega)$, then there will be also a maximum for the reflected field only if it holds $\left|\frac{d^{2}\left|E^{(i)}\left(\omega_{0}^{(i)}\right)\right|^{2}}{d \omega^{2}}\right|_{\omega_{0}^{(i)}} R\left(\omega_{0}^{(i)}\right)>\left|E^{(i)}\left(\omega_{0}^{(i)}\right)\right|^{2}\left|\frac{d^{2} R(\omega)}{d \omega^{2}}\right|_{\omega_{0}^{(i)}}$

[12] G. Bönsch and E. Potulski, Metrologia, 35, 133-139, (1998).

[13] An evaluation of $d^{2} \omega(k) / d k^{2}$ in eqq 9 does not require the knowledge of the function $\omega(k)$ i.e. the inverse function of the dispersion relation $k(\omega)=n(\omega) \omega / c$. In fact, it is possible to note that if $k(\omega)$ is invertible around a frequency $\tilde{\omega}$ such that $\tilde{k}=k(\tilde{\omega})$, then, after some 
algebra, we have

$$
\left(\frac{d^{2} \omega}{d k^{2}}\right)_{\tilde{k}}=-\left(\frac{d^{2} k}{d \omega^{2}}\right)_{\tilde{\omega}}\left[\left(\frac{d k}{d \omega}\right)_{\tilde{\omega}}\right]^{-3}
$$

[14] In the cited example, summarized by fig 2, we have:

$$
\begin{aligned}
& \omega_{0}^{(t)}=3,3061 \times 10^{15} \mathrm{rad} / \mathrm{s}, \\
& \omega_{0}^{(r)}=3,4527 \times 10^{15} \mathrm{rad} / \mathrm{s}, \\
& d^{2} \omega /\left.d k^{2}\right|_{k_{0}^{(i)}}=-8,42 \times 10^{-4} \mathrm{~m}^{2} / \mathrm{s} \text { and } \\
& v_{g}^{(i)}=299.701 .445 \mathrm{~m} / \mathrm{s} .
\end{aligned}
$$

[15] F. S. Crawford, Waves, McGraw-Hill (1968). 


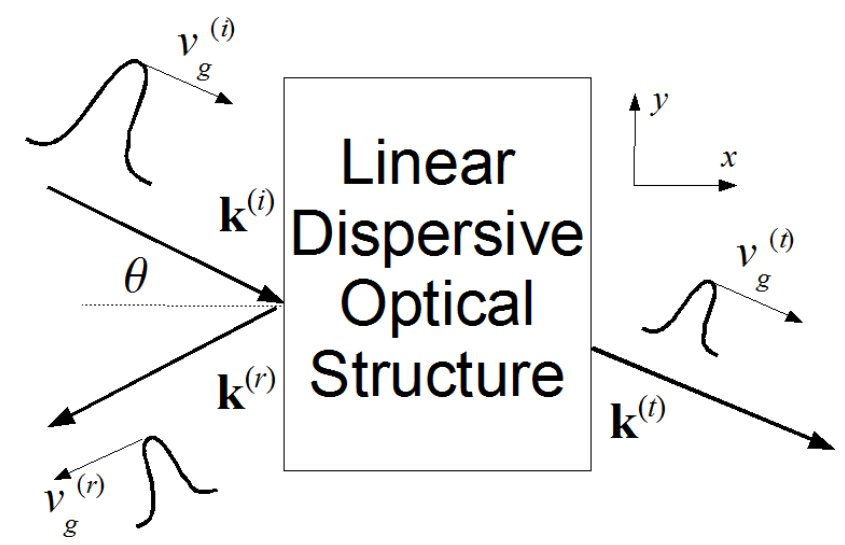

FIG. 1: An optical beam incident over a linear dispersive and finite optical structure (such as a Beam Splitter or a PBG or a Lens etc.) is represented. The transmitted and reflected beams are schematically depicted as two other smaller wave packets, traveling in different directions. 

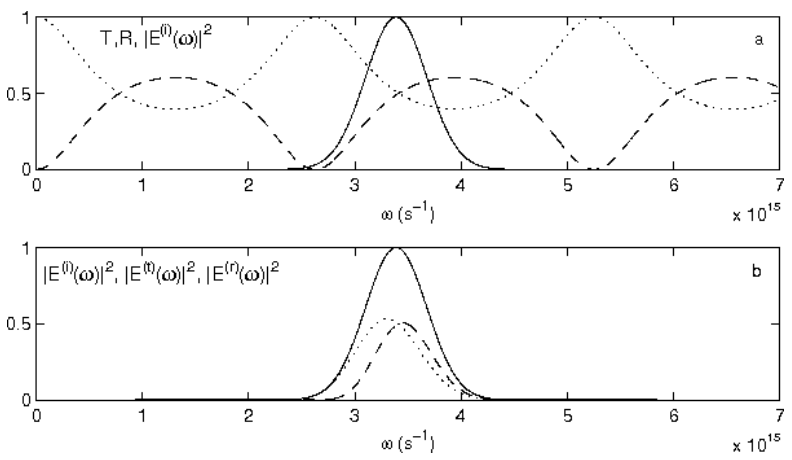

FIG. 2: Transmitted (dotted line) and reflected (dashed line) field spectra originating from a rectangularly symmetric slab of thickness $0.3 \mu \mathrm{m}$ and refractive index $n=1,5$. The incident field (continuous line) has an angle of incidence $\theta_{i}=65^{\circ}$. 